\title{
Activation of Pedunculopontine Glutamate Neurons Is Reinforcing
}

\author{
Ji Hoon Yoo, ${ }^{1}$ Vivien Zell, ${ }^{1}$ Johnathan Wu, ${ }^{1}$ Cindy Punta, ${ }^{1}$ Nivedita Ramajayam, ${ }^{1}$ Xinyi Shen, ${ }^{1}$ Lauren Faget, ${ }^{1}$ \\ Varoth Lilascharoen, ${ }^{2}$ Byung Kook Lim, ${ }^{2}$ and ${ }^{\circ}$ Thomas S. Hnasko ${ }^{1}$ \\ ${ }^{1}$ Department of Neurosciences and ${ }^{2}$ Department of Biological Sciences, University of California, San Diego, La Jolla, California 92093
}

\begin{abstract}
Dopamine transmission from midbrain ventral tegmental area (VTA) neurons underlies behavioral processes related to motivation and drug addiction. The pedunculopontine tegmental nucleus (PPTg) is a brainstem nucleus containing glutamate-, acetylcholine-, and GABA-releasing neurons with connections to basal ganglia and limbic brain regions. Here we investigated the role of PPTg glutamate neurons in reinforcement, with an emphasis on their projections to VTA dopamine neurons. We used cell-type-specific anterograde tracing and optogenetic methods to selectively label and manipulate glutamate projections from PPTg neurons in mice. We used anatomical, electrophysiological, and behavioral assays to determine their patterns of connectivity and ascribe functional roles in reinforcement. We found that photoactivation of PPTg glutamate cell bodies could serve as a direct positive reinforcer on intracranial selfphotostimulation assays. Further, PPTg glutamate neurons directly innervate VTA; photostimulation of this pathway preferentially excites VTA dopamine neurons and is sufficient to induce behavioral reinforcement. These results demonstrate that ascending PPTg glutamate projections can drive motivated behavior, and PPTg to VTA synapses may represent an important target relevant to drug addiction and other mental health disorders.
\end{abstract}

Key words: dopamine; glutamate; optogenetics; pedunculopontine; reward; ventral tegmental area

\section{Significance Statement}

Uncovering brain circuits underlying reward-seeking is an important step toward understanding the circuit bases of drug addiction and other psychiatric disorders. The dopaminergic system emanating from the ventral tegmental area (VTA) plays a key role in regulating reward-seeking behaviors. We used optogenetics to demonstrate that the pedunculopontine tegmental nucleus sends glutamatergic projections to VTA dopamine neurons, and that stimulation of this circuit promotes behavioral reinforcement. The findings support a critical role for pedunculopontine tegmental nucleus glutamate neurotransmission in modulating VTA dopamine neuron activity and behavioral reinforcement.

\section{Introduction}

The pedunculopontine tegmental nucleus (PPTg) is a brainstem nucleus, delineated by its cholinergic neuron population and implicated in several important brain functions, including locomotion, sleep, cognition, and arousal (Ryczko and Dubuc, 2013; Gut

\footnotetext{
Received Oct. 4, 2016; accepted 0ct. 29, 2016

Author contributions: J.H.Y., V.Z., and T.S.H. designed research; J.H.Y., V.Z., J.W., C.P., N.R., and X.S. performed research; L.F., V.L., and B.K.L. contributed unpublished reagents/analytic tools; J.H.Y., V.Z., V.L., B.K.L., and T.S.H. analyzed data; J.H.Y., V.Z., and T.S.H. wrote the paper.

This work was supported by Tobacco-related disease research program Grant 24RT-0026 to T.S.H. and Grant 22FT-0063 to J.H.Y., National Institutes of Health Grant R01DA036612 to T.S.H. and Grant R01MH108594 to B.K.L., National Institute on Drug Abuse-Institut National de la Santé et de la Recherche Médicale postdoctoral fellowship to V.Z., the Brain Research Foundation to L.F., and the Ananda Mahidol fellowship to V.L. We thank Alexander Johnson, Reed Ressler, and Mohammad Ali Shenasa for technical assistance.

The authors declare no competing financial interests.

Correspondence should be addressed to Dr. Thomas S. Hnasko, Department of Neurosciences, University of California, San Diego, La Jolla, CA 92093. E-mail: thnasko@ucsd.edu.

DOI:10.1523/JNEUROSCI.3082-16.2016

Copyright $@ 2017$ the authors $\quad 0270-6474 / 17 / 370038-09 \$ 15.00 / 0$
}

and Winn, 2016). Ascending projections from the PPTg target nuclei in the basal ganglia and thalamus and contribute to motor and nonmotor behavioral outputs dependent on basal ganglia drive (Mena-Segovia et al., 2004; Martinez-Gonzalez et al., 2011). For example, one recent study demonstrated that glutamate neurons in the PPTg and throughout the mesencephalic locomotor region encode locomotion and speed and are modulated by basal ganglia activity (Roseberry et al., 2016).

The PPTg also projects to both dopamine and nondopamine neurons in the substantia nigra pars compacta (SNc) and ventral tegmental area (VTA) (Charara et al., 1996; Faget et al., 2016). PPTg activity can evoke dopamine release in the nucleus accumbens (Forster and Blaha, 2003), drive dopamine neuron burst firing (Lokwan et al., 1999; Floresco et al., 2003), and encode reward-related signals posited to be of importance for reinforcement learning (Pan and Hyland, 2005; Okada et al., 2009; Norton et al., 2011). PPTg lesions can disrupt stimulus-reward learning (Inglis et al., 2000) and blunt the incentive properties of opiates 
and psychostimulants (Bechara and van der Kooy, 1989; Olmstead et al., 1998). Recent work targeting PPTg cholinergic projections to the VTA demonstrate that these cells synapse on to both dopamine and nondopamine neurons and can bidirectionally modulate behavioral reinforcement (Dautan et al., 2016; Xiao et al., 2016). These results demonstrate the important role for the PPTg in regulating fundamental behaviors, and deciphering how specific cell type and projection target elements contribute to these behaviors has important implications for understanding basic processes underlying reward seeking, movement, and mental illness.

Although defined by its cholinergic population, the PPTg is neurochemically heterogeneous consisting also of GABAergic and glutamatergic cell types (Wang and Morales, 2009; Martinez-Gonzalez et al., 2011). Glutamate inputs from several brain areas synapse on to VTA to directly or indirectly modulate dopamine neuron activity, and plasticity in these circuits has been linked to the development of addiction (Grace et al., 2007; Geisler and Wise, 2008; Lüscher and Malenka, 2011). The ability of PPTg inputs to directly excite and control conditioned responses of dopamine and nondopamine neurons suggests a potentially important role for the glutamatergic portion of this heterogeneous circuit (Futami et al., 1995; Yau et al., 2016). However, cell-type-specific approaches to delineate the projection targets or functions of PPTg glutamate neuron activation have not been reported. Here we address this question using viral mediated tracing strategies and optogenetics to demonstrate that PPTg glutamate neurons and their projections to VTA can potently drive reinforcement.

\section{Materials and Methods}

Animals. Mice were used in accordance with protocols approved by the University of California San Diego Institutional Animal Care and Use Committee. VGLUT2- $\left(\right.$ Slc17a6 $6^{\text {tm2 (cre)Lowl }}$, stock \#016963) IRES-Cre knock-in mice (Vong et al., 2011) were obtained from The Jackson Laboratory and then bred in house. Mice were fully ( $>10$ generations) backcrossed on to C57BL/6, both sexes were used across experiments, and mice were used for behavior between the ages of 10 and 18 weeks. Mice were group housed and maintained on a $12 \mathrm{~h}$ light-dark cycle with food and water available ad libitum unless noted.

Stereotactic surgery. Mice ( $>4$ weeks) were anesthetized with isoflurane and placed in a stereotaxic frame (David Kopf Instruments). For microinfusion of virus, a custom-made 30-guage stainless injector was used to unilaterally infuse AAV1-EF1 $\alpha$-DIO-Channelrhodopsin-2 (ChR2):mCherry (100-300 nl, $\sim 2 \times 10^{12}$ genomes/ml, UNC gene therapy center), AAV2-EF1 $\alpha$-DIO:mCherry $\left(100-300 \mathrm{nl}, \sim 2 \times 10^{12}\right.$ genomes/ml, UNC gene therapy center), or AAVDJ-Syn-DIO-mRubyT2A-Synaptophysin:EGFP (200 nl, BK Lim) into the PPTg $(x=-1.1$, $y=-4.48, z=-3.75$; $\mathrm{mm}$ relative to bregma) at $100 \mathrm{nl} / \mathrm{min}$ using a micropump (WPI UltraMicroPump). For behavioral experiments, custom-built optic fibers $(200-\mu \mathrm{m}$ multimode optical fiber, Thorlabs) were implanted dorsal to PPTg $(x=-1.1, y=-4.48, z=-3.5)$ or VTA $(x=-0.6, y=-3.4, z=-4.0)$. Following viral vector injection and optic fiber implantation, mice were allowed to recover $>3$ weeks before behavior and/or perfusion for histological processing.

Electrophysiological recordings from acute adult brain slices. Adult mice (6-11 weeks) were deeply anesthetized with pentobarbital $(200 \mathrm{mg} / \mathrm{kg}$ i.p.; Virbac) and perfused intracardially with $10 \mathrm{ml}$ ice-cold sucrosebased ACSF containing the following (in $\mathrm{mm}$ ): 75 sucrose, $87 \mathrm{NaCl}, 2.5$ $\mathrm{KCl}, 7 \mathrm{MgCl}_{2}, 0.5 \mathrm{CaCl}_{2}, 1.25 \mathrm{NaH}_{2} \mathrm{PO}_{4}, 25 \mathrm{NaHCO}_{3}$, and continuously bubbled with carbogen $\left(95 \% \mathrm{O}_{2}-5 \% \mathrm{CO}_{2}\right)$. Brains were extracted and $200-\mu \mathrm{m}$ coronal slices were cut in sucrose-ACSF using a Vibratome (vt1200, Leica). Slices were transferred to a perfusion chamber containing ACSF at $31^{\circ} \mathrm{C}$ (in mM) as follows: $126 \mathrm{NaCl}, 2.5 \mathrm{KCl}, 1.2 \mathrm{MgCl}_{2}, 2.4$ $\mathrm{CaCl}_{2}, 1.4 \mathrm{NaH}_{2} \mathrm{PO}_{4}, 25 \mathrm{NaHCO}_{3}, 11$ glucose, continuously bubbled with carbogen. After at least $45 \mathrm{~min}$, slices were transferred to a recording chamber continuously perfused with ACSF (2-3 ml/min) and maintained at $29^{\circ} \mathrm{C}-31^{\circ} \mathrm{C}$ using an inline heater. Patch pipettes $(3.5-5.5 \mathrm{M} \Omega$ ) were pulled from borosilicate glass (King Precision Glass) and filled with internal recording solution containing the following (in $\mathrm{mM}$ ): 120 $\mathrm{CsCH}_{3} \mathrm{SO}_{3}, 20$ HEPES, 0.4 EGTA, $2.8 \mathrm{NaCl}, 5$ TEA, $2.5 \mathrm{Mg}$-ATP, 0.25 $\mathrm{Na}-\mathrm{GTP}$, at pH 7.25 and $285 \mathrm{mOsm}$; a subset of recordings included $0.1 \%$ Lucifer yellow (LY) (Sigma).

mCherry-labeled VGLUT2 ${ }^{+}$PPTg terminals were visualized by epifluorescence, and visually guided patch recordings were made from VTA cells (located medial to the medial terminal nucleus of the accessory optic system and/or immediately dorsal to the interpeduncular nucleus) using infrared-differential interference contrast illumination (Axiocam MRm, Examiner.A1, Zeiss). ChR2 was activated by flashing blue light (5 ms pulse width to induce postsynaptic currents in whole-cell configuration and $5 \mathrm{~ms}$ pulse width in $40-\mathrm{Hz}$ trains for $5 \mathrm{~s}$ in cell-attached recordings) through the light path of the microscope using a light-emitting diode (UHP-LED460, Prizmatix) under computer control. EPSCs were recorded in whole-cell voltage clamp (Multiclamp 700B amplifier), filtered at $2 \mathrm{kHz}$, digitized at $10 \mathrm{kHz}$ (Digidata 1550) and collected on-line using Clampex 10.6 software (Molecular Devices). Series resistance and capacitance were electronically compensated before recordings. Estimated liquid-junction potential was $12 \mathrm{mV}$ and left uncorrected. Series resistance was monitored and cells that showed $>25 \%$ change during recordings were considered unstable and discarded. Neurons were held in voltage-clamp at $-70 \mathrm{mV}$ to record AMPAR EPSCs in whole-cell configuration. For whole-cell voltage-clamp recordings, single-pulse (5 ms) photostimuli were applied every $55 \mathrm{~s}$ and 10 photo-evoked currents were averaged per neuron per condition; current sizes were calculated by using peak amplitude from baseline. For cell-attached studies on firing rate, photostimuli trains ( 1 or $5 \mathrm{~s}$ ) were delivered every $45 \mathrm{~s}$ and 3 responses averaged per neuron. Action potential frequency was averaged over the 1 or $5 \mathrm{~s}$ before, during, and after the stimulation train. DMSO containing DNQX (Sigma) was diluted 1000-fold in ACSF and bath applied at final concentration of $10 \mu \mathrm{M}$.

Instrumental nosepoke assay. Mice were tested in operant chambers (Med Associates) for $45 \mathrm{~min}$ and nosepoke responses were recorded by MedPC IV software. Before starting the test, each animal was connected to a patch cable (50 $\mu \mathrm{m}$ multimode optic fiber, Thorlabs). A brief tone (2 $\mathrm{kHz}, 0.5 \mathrm{~s}$ ) was generated at the beginning of each daily session. A sucrose pellet (Bio-Serv, F0071) for each nosepoke hole was provided as bait at the beginning of each session to encourage nosepokes. Two nosepoke holes were available: a nosepoke on the side predesignated as active was programmed to produce a brief tone $(2 \mathrm{kHz}, 0.5 \mathrm{~s})$ and deliver 10 or 15 pulses (5-ms pulse width) at $80 \mathrm{~mW} / \mathrm{mm}^{2}(\sim 10 \mathrm{~mW})$ from the $200-\mu \mathrm{m}$ core implanted optic fiber tip via Arduino control, whereas nosepoke to the inactive side produced the tone without triggering the laser $(473 \mathrm{~nm}$ DPSS Laser, OEM Laser). Animals were on a restricted feeding schedule and provided food ad libitum for $3 \mathrm{~h}$ daily after each daily session. Occasionally mice made zero nospokes at either active ( 1 control for cell body stimulation and 1 control for terminal stimulation) or inactive holes (3 control and 9 ChR2 for cell body stimulation; 1 control and 4 ChR2 for terminal stimulation) across all sessions; to calculate preference scores, one nosepoke was thus added to both active and inactive counts. A digital power meter (PM100D/S121C, Thorlabs) was used to measure the light output of the DPSS blue laser (473 nm, OEM Laser). In the 5-choice experiments, the 2 individual nosepoke holes were replaced with a 5-nosepoke-hole wall and each hole was coupled to different frequencies of photostimulation (none, $10,20,30$, or $40 \mathrm{~Hz}$; $0.5 \mathrm{~s}$ duration; 60 min sessions).

Two bottle choice test. Water was removed overnight and experiments were performed the following day. Mice were supplied with water for $3 \mathrm{~h}$ daily at the end of each session. On day 1 (baseline), mice were placed in standard operant conditioning chambers equipped with two identical water-filled sippers (Med Associates). Licks were recorded for $45 \mathrm{~min}$ using contact lickometers (Med Associates). On subsequent days, one side was designated active and 5 licks at the active sipper triggered 10 pulses (5-ms pulse width, $10 \mathrm{~mW}, 20 \mathrm{~Hz}$ ) of photostimulation controlled by Med-PC IV package. Licks on the inactive sipper had no effect but were recorded. The active sipper was counterbalanced across animals. On days $5-8$, the water in the inactive sipper was replaced with escalating concentrations of sucrose $(1 \%-8 \%)$. 


\section{A}
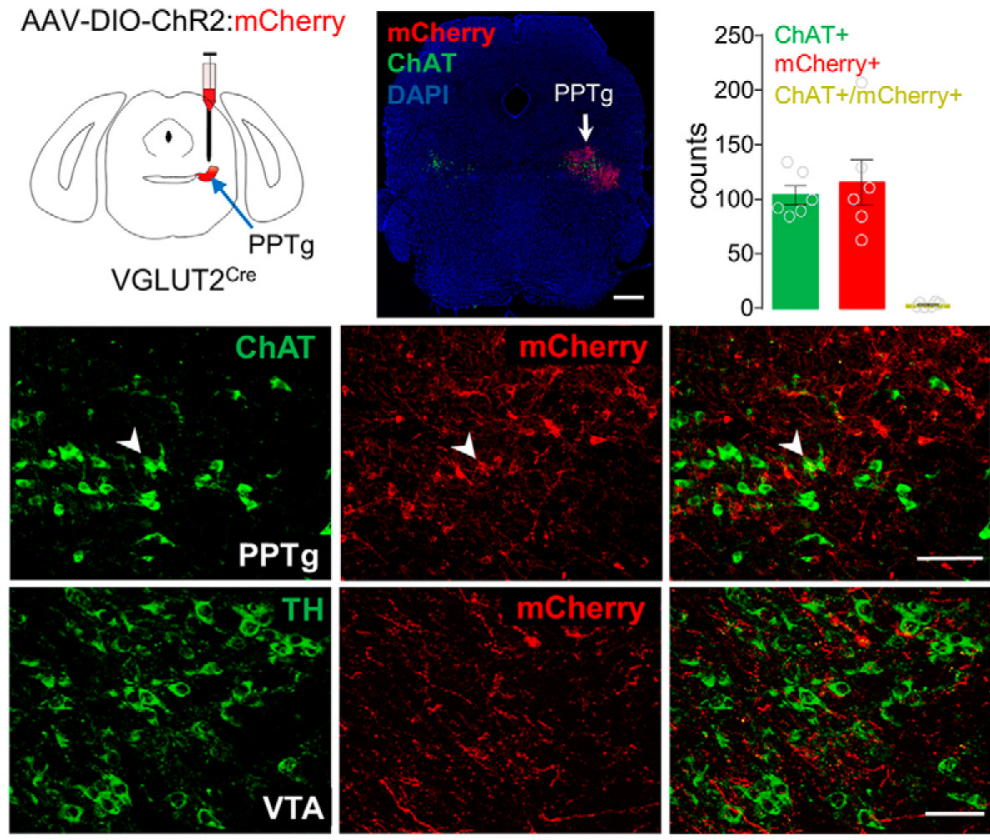

B
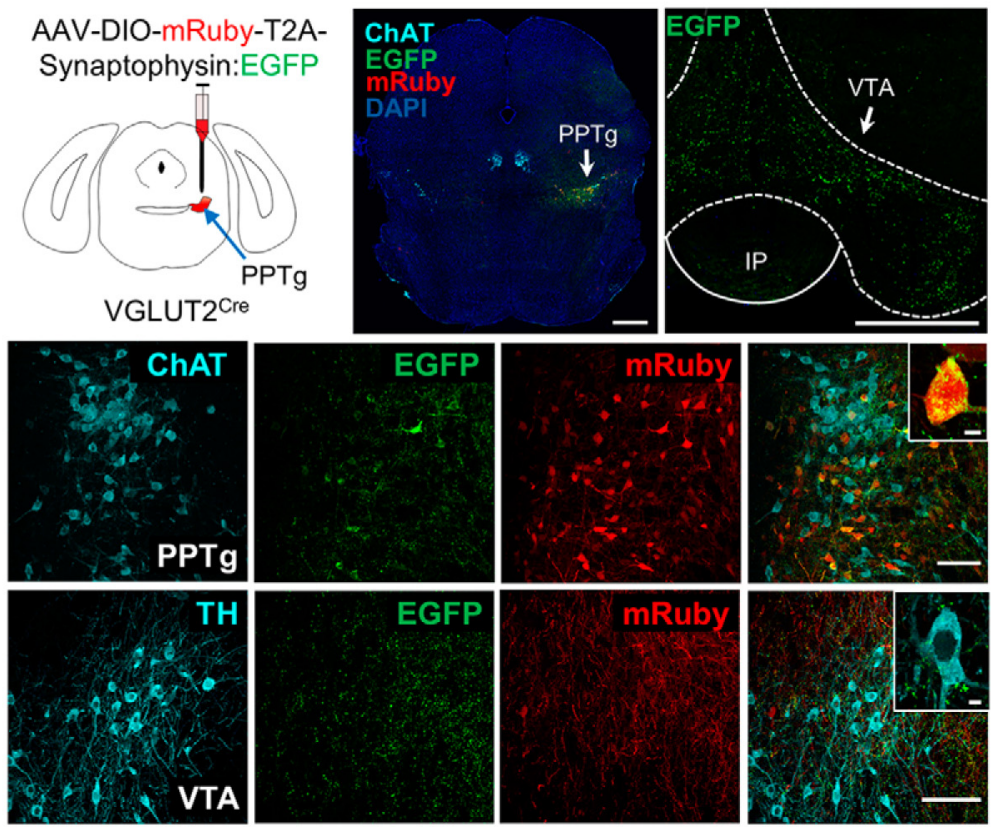

Figure 1. PPTg glutamate neurons project to VTA. PPTg and VTA area were delineated by ChAT and TH immunostaining respectively. $\boldsymbol{A}$, Top, Schematic for labeling VGLUT2 ${ }^{+}$neurons in the PPTg by microinjection of AAV-DI0-ChR2:mCherry into the PPTg of VGLUT2 ${ }^{\text {Cre }}$ mice (left). ChR2:mCherry-expressing soma in the PPTg (middle). Scale bar, $500 \mu \mathrm{m}$. Cell counts of of ChAT ${ }^{+}$ ChR2:mCherry ${ }^{+}$, and ChAT ${ }^{+}$and ChR2:mCherry ${ }^{+}$dual-labeled cells in PPTg (right; $n=6$ mice). Middle row, ChR2:mCherry labeling in VGLUT2 ${ }^{\text {Cre }}$ cells of the PPTg, ChR2:mCherry/ChAT double-labeled cell (arrowhead). Scale bar, $100 \mu \mathrm{m}$. Bottom row, ChR2:mCherry ${ }^{+}$fibers abundant near $\mathrm{TH}^{+}$dopamine neurons in VTA. Scale bar, $50 \mu \mathrm{m}$. $\boldsymbol{B}$, Top, Schematic for microinjection of AAV-DI0-mRuby-T2A-Synaptophysin:EGFP into the PPTg of VGLUT2 ${ }^{\text {Cre }}$ mice (left). mRuby-Synaptophysin:EGFP-expressing soma in the PPTg (middle). Scale bar, $500 \mu \mathrm{m}$. Synaptophysin:EGFP-expressing terminal fibers in the VTA (right). IP, interpeduncular nucleus. Scale bar, $500 \mu \mathrm{m}$. Middle row, mRuby-labeled cells and Synaptophysin:EGFP in the PPTg. Scale bar, $100 \mu \mathrm{m}$. Inset, Highmagnification image of local glutmate terminals in the PPTg. Scale bar, $5 \mu \mathrm{m}$. Bottom row, mRuby-labeled fibers and Synaptophysin:EGFP puncta in VTA-projecting PPTg terminals. Scale bar, $100 \mu \mathrm{m}$. Inset, High-magnification image. Scale bar, $5 \mu \mathrm{m}$.

Immunohistochemistry. Mice were anesthetized through an intraperitoneal injection of ketamine (Pfizer, $10 \mathrm{mg} / \mathrm{kg}$ ) and xylazine (Lloyd, 2 $\mathrm{mg} / \mathrm{kg}$ ). Animals were then transcardially perfused with ice-cold PBS followed by $4 \%$ PFA. Brains were incubated in 4\% PFA overnight, transferred to $30 \%$ sucrose solution for 48 to $70 \mathrm{~h}$ until sunk, frozen in chilled isopentane, cut to $30 \mu \mathrm{m}$ thickness on a cryostat (CM3050S, Leica), and collected in PBS containing $0.01 \%$ sodium azide. For immunohisto- chemistry, brain sections were washed three times ( $5 \mathrm{~min}$ ) in PBS, three times $(5 \mathrm{~min})$ in PBS containing $0.2 \%$ Triton X-100 (PBS-Tx), and blocked in PBS-Tx containing 4\% normal donkey serum (NDS) for $1 \mathrm{~h}$ at room temperature. Free-floating sections were then incubated in primary antibody against $\mathrm{TH}$ (rabbit anti-TH, 1:2000, Millipore AB152), GFP (rabbit anti-GFP, 1:2000, Invitrogen A11122), ChAT (goat anti-ChAT, 1:400, Millipore $\mathrm{AB} 144 \mathrm{P}$ ), and/or c-Fos (rabbit anti-cFos, 1:7000, Calbiochem PC38) at $4^{\circ} \mathrm{C}$ overnight. Sections were washed three times $(10 \mathrm{~min})$ in blocking solution (PBS-Tx), then incubated with secondary antibodies (donkey anti-goat or anti-rabbit conjugated to Alexa-488 or Alexa-647 fluorescent dyes, $5 \mu \mathrm{g} / \mathrm{ml}$, Jackson ImmunoResearch Laboratories) for $2 \mathrm{~h}$ at room temperature. Slices were rinsed three times (10 $\mathrm{min})$ with $\mathrm{PBS}$, before mounting onto slides with Flouromount-G mounting medium (Southern Biotech) \pm DAPI (Roche, $0.5 \mu \mathrm{g} / \mathrm{ml}$ ). Mice used for c-Fos counts were subjected to the two-nosepoke test for $3 \mathrm{~d}$, and brains were extracted $90 \mathrm{~min}$ after the start of testing on day 3. Quantification of ${\mathrm{c}-\mathrm{Fos}^{+}}^{+} \mathrm{nu}-$ clei was conducted by sampling 2 sections through the PPTg (4.24-4.72 $\mathrm{mm}$ posterior to bregma) and 5 sections through the VTA (2.92-3.88 $\mathrm{mm}$ posterior to bregma) of each animal by an investigator blind to treatment.

To verify whether the recorded cells were dopaminergic neurons, we performed post hoc immunostaining for $\mathrm{TH}$ on a subset of $200-\mu \mathrm{m}$ slices used for electrophysiology. Slices were postfixed with 4\% PFA overnight and processed for $\mathrm{TH}$ immunohistochemistry as described above, except: primary antibody, rabbit anti-TH, 1:1000, $48 \mathrm{~h}$ at $4^{\circ} \mathrm{C}$; secondary antibody incubation for $24 \mathrm{~h}$ at $4^{\circ} \mathrm{C}$.

Images were acquired using a Leica SP5 confocal microscope, an Olympus VS-120 Virtual Slide Scanning Microscope, an Olympus BX53 epifluorescence microscope, or Zeiss Axio Observer equipped with ApoTome. Image processing and cell counting were performed using ImageJ (National Institutes of Health) and/or Zeiss Zen software (Zeiss).

Statistical analyses. Statistical analysis was performed using Graphpad or Statistica. Behavioral and all other data were evaluated using two-way repeated measures ANOVA, and all data are expressed as mean \pm SEM unless otherwise indicated.

\section{Results}

\section{Glutamate-containing neurons in the} PPTg project to the VTA

To identify whether glutamate neurons in the PPTg project to the VTA, we stereotactically infused an Adeno-associated viral vector that Cre-dependently expresses ChR2 fused with a red fluorescent reporter (AAV-DIO-ChR2:mCherry) (Fig. $1 A$ ) into the PPTg of Slc17a6 ${ }^{\text {Cre }}$ (VGLUT2 ${ }^{\mathrm{Cre}}$ ) mice. Fewer than $4 \%$ of ChR2:mCherry cells colabeled for the cholinergic marker ChAT, suggesting that this manipulation targets a separate population. We identified ChR2:mCherry containing axonal fibers from VGLUT2 ${ }^{\mathrm{Cre}}$-labeled PPTg cells in the VTA (Fig. $1 A$ ) and 
A

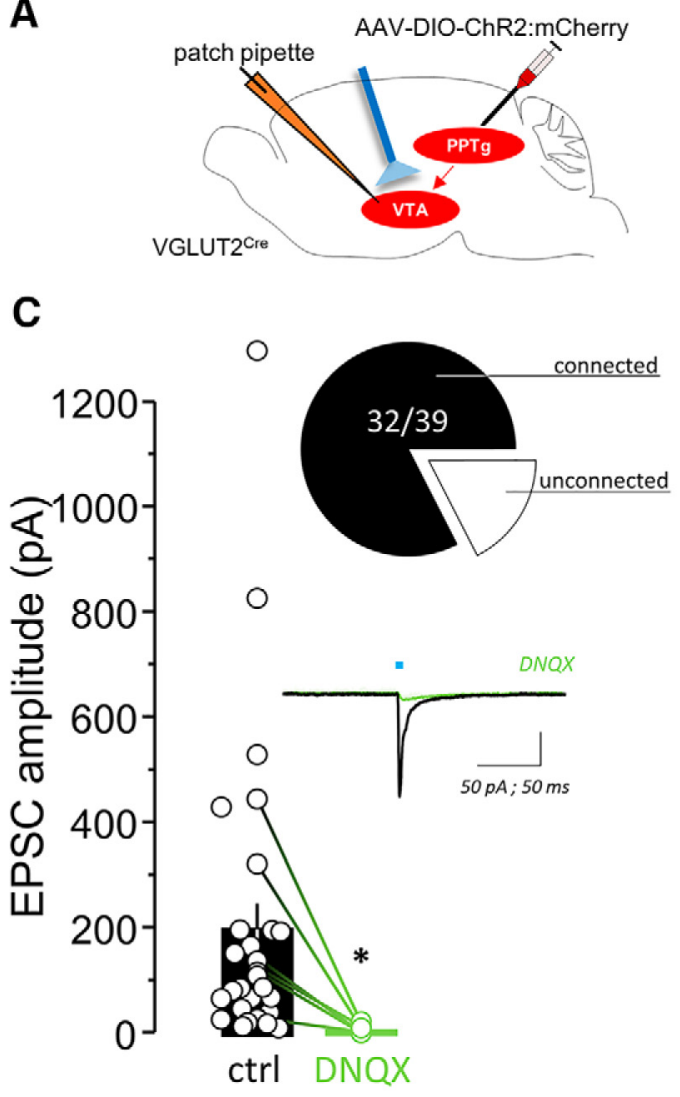

B

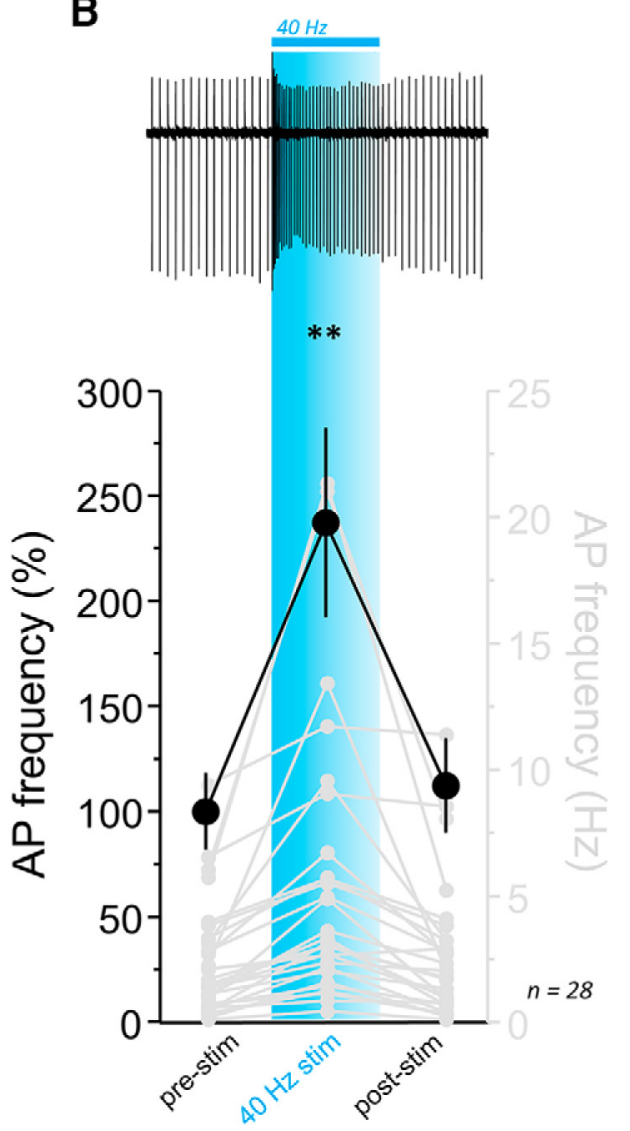

Figure2. Optogenetic stimulation of PPTg glutamate terminals in the VTAis excitatory. $\boldsymbol{A}$, Schematicillustrating experimental approach. $\boldsymbol{B}$, An example trace (top) from cell-attached recording of VTA neuron identified proximal to ChR2:mCherry fluorescence from VGLUT2 ${ }^{+}$PPTg fibers show that photostimulation $(40 \mathrm{~Hz}, 5 \mathrm{~s})$ increases firing rate. Bottom, Mean increase in firing rate (black) and the firing rates of individual units (gray) in 5 s bins before, during, and after photostimulation. The $40 \mathrm{~Hz}$ photostimulation produced a significantincrease in firing rate from $2.47 \pm 0.46 \mathrm{~Hz}$ t0 $5.96 \pm 1.12 \mathrm{~Hz}$. ${ }^{* *} p<0.01$ (one-way ANOVA, $F_{(2,72)}=6.28$; followed by Tukey-Kramer post hoc test). C, Voltage-clamp recordings revealed that most VTA cells showed EPS(s to single-pulse stimulation. Graph represents mean EPSC size. DNQX significantly reduced size. ${ }^{*} p<0.05$ (paired $t$ test). Inset, Example trace to blue light pulse (blue) before (black) and after (green) DNQX application.

several other areas, including SNc, subthalamic nucleus, thalamus, and lateral hypothalamus (data not shown). To test whether the fibers in VTA reflect functional synaptic release sites, we injected a Cre-dependent bicistronic AAV to express both mRuby in the cytosol and a Synaptophysin:EGFP fusion protein to label presynaptic terminals (AAV-DIO-mRuby-T2A-Synaptophysin: EGFP). Expression of mRuby was detected in both PPTg soma and terminals in the VTA (Fig. $1 B$ ), similar to ChR2:mCherry (Fig. 1A). Green fluorescent puncta indicative of Synaptophysin: EGFP were also present in VTA, suggesting that PPTg glutamate neurons make functional glutamatergic contacts.

\section{Optogenetic stimulation evokes glutamate release from PPTg to VTA dopamine neurons}

To examine the synaptic output of PPTg glutamate neurons in the VTA, we performed patch-clamp recordings on acute brain slices of VGLUT2 ${ }^{\text {Cre }}$ mice injected with AAV-DIO-ChR2: mCherry into the PPTg (Fig. 2A). Allowing at least 3 weeks after viral injection, we made acute brain slices for electrophysiological recordings in the VTA while selectively photostimulating PPTg glutamate terminals. Using cell-attached recordings, we found that high-frequency optogenetic stimulation $(40 \mathrm{~Hz})$ of PPTg glutamate terminals produced a significant increase in VTA neuron firing rates (Fig. $2 B$ ). In whole-cell voltage clamp, delivery of single light pulses elicited EPSCs in $82 \%$ (32 of 39) of neurons recorded in VTA (Fig. 2C). To determine the neurotransmitter identity of the postsynaptic cell, a subset of recordings included LY in the recording pipette to allow for post hoc identification of dopamine neurons by immunohistochemistry against the dopamine marker TH (Fig. $3 A$ ). Of the 22 recorded cells identified in this way, TH was detected in 13, whereas 9 were TH negative. Although fast EPSCs $(<3 \mathrm{~ms}$ delay) were detected in both dopamine $\left(\mathrm{TH}^{+}, \mathrm{LY}^{+}\right)$and putative nondopamine $\left(\mathrm{TH}^{-}, \mathrm{LY}^{+}\right)$neurons, EPSCs were significantly larger in the neurons verified as $\mathrm{TH}^{+}$(Fig. $3 B$ ). Cell-attached recording from $\mathrm{TH}^{+}$and $\mathrm{TH}^{-}$neurons showed a significant increase in firing rate during high-frequency photostimulation of PPTg terminals (Fig. 3C).

We next assessed functional activation in vivo by immunostaining for the immediate early gene c-Fos. Following the in vivo optogenetic stimulation of PPTg neurons, we observed a $>4$-fold increase in the number of nuclei labeled with c-Fos in the PPTg of ChR2:mCherry-expressing mice compared with mCherryexpressing controls (Fig. 4A). We also assessed the VTA of these mice and found that optogenetic activation of PPTg evoked a $>3$-fold increase in the number VTA neurons labeling for c-Fos (Fig. $4 B, D$ ). The number of c-Fos-labeled nuclei was increased in both $\mathrm{TH}^{+}$and $\mathrm{TH}^{-}$neurons (Fig. $4 C$ ), whereas the fraction of c-Fos-labeled cells that colabeled for $\mathrm{TH}$ was $8 \%$ in the mCherry control group compared with $31 \%$ in the ChR2 group. Together, 
these results indicate that PPTg glutamate neurons target both dopamine and nondopamine neurons in the VTA, but their impact may be functionally greater on the dopaminergic population.

\section{Optogenetic stimulation of PPTg} glutamate cell bodies or their terminals in VTA can drive

\section{behavioral reinforcement}

To determine whether activation of genetically defined PPTg glutamate neurons can serve as a reinforcer, we used in vivo optogenetic stimulation. We used the conditional virus approach described above (Fig. 1A) to selectively express ChR2:mCherry or control (mCherry) in PPTg neurons of VGLUT2 ${ }^{\text {Cre }}$ mice and to deliver light to behaving animals we implanted optic fibers over the PPTg (Fig. $5 A$ ). At least 3 weeks after surgery, ChR2or mCherry-expressing (control) animals were placed in operant chambers equipped with two nosepoke holes. Nosepokes on the active hole led to photostimulation, whereas the inactive hole produced none (Fig. 5B). ChR2expressing VGLUT2 ${ }^{\mathrm{Cr}}$ mice showed a marked preference for and made significantly more active nosepokes than control mice at 20 and $30 \mathrm{~Hz}$ (Fig. 5B). In a separate assay, we tested mice over $3 \mathrm{~d}$ on a 5-nosepoke discrimination task comparing $0-40 \mathrm{~Hz}$ stimulation (Fig. 5C). Mice were able to discriminate and showed a preference for nosepoke holes coupled to stimulus frequencies $>20 \mathrm{~Hz}$. To extend these results, we tested water-restricted mice in a two-bottle choice assay using operant chambers equipped with contact lickometers (Fig. 5D). On baseline day (day 1), mice were provided access to two water-filled bottles, licks were recorded, and sippers then designated as active and inactive to balance intrinsic preference ratios within treatment groups. On days $2-4$, one bottle was coupled to photostimulation (active) and the other not (inactive). ChR2-expressing mice showed a preference for and made more licks on the sipper coupled to laser activation of PPTg glutamate neurons, whereas control mice showed no preference for either side. On days 5-8, water in the inactive side was replaced with increasing concentrations of sucrose solution. Control animals developed a preference for the sucrose-filled sipper, whereas ChR2-expressing mice preferred the sipper coupled to laser stimulation over even the highest concentrations of sucrose tested (Fig. 5D). Optic fiber placements were verified post hoc on sections immunostained for ChAT to delineate PPTg (Fig. 5A,E).

To test whether the projections from PPTg to VTA may subserve these effects, we repeated these experiments, but in animals with implants dorsal to VTA rather than PPTg (Fig. 6A). We found that photstimulation of PPTg glutamate terminals in the VTA could serve as an operant reinforcer in the 2-nosepoke assay
B

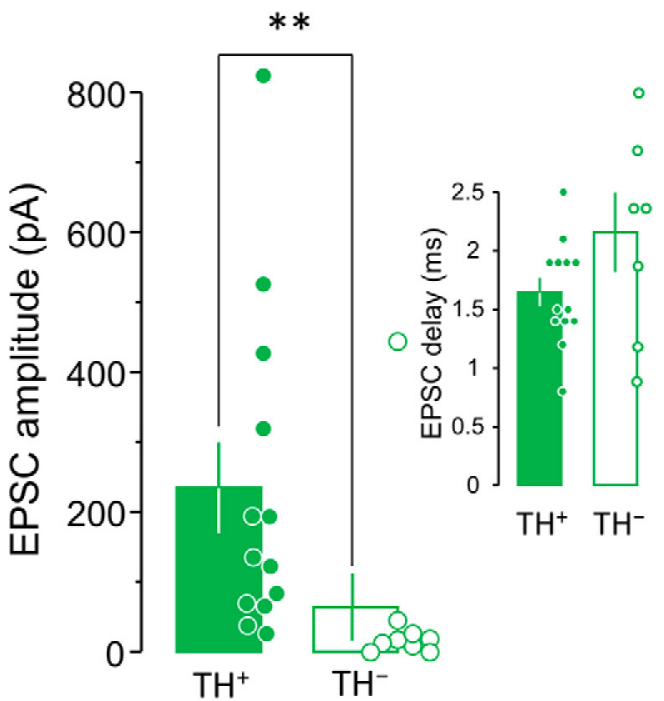

Figure 3. The PPTg provides greater excitation on to VTA dopamine versus nondopamine neurons. $A$, Neurons filled with LY

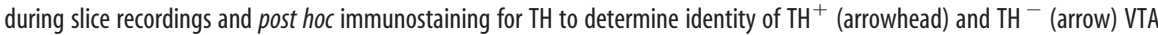
mean \pm SEM. C, Photostimulation $(40 \mathrm{~Hz})$ of PPTg terminals increased firing rates of $\mathrm{TH}^{+}$VTA neurons and $\mathrm{TH}^{-}$VTA lation $F_{(2,30)}=22.9, p<0.001$; stimulation $\times$ cell type $\left.F_{(2,30)}=8.5, p<0.01\right)$. Right, Actual firing rates (stimulation $F_{(1,15)}=$ $46.8, p<0.001)$. Data are mean \pm SEM.

(Fig. 6B), and in the two-bottle choice assay where stimulation was again preferred over sucrose (Fig. 6C). Optic fiber placements were again verified post hoc by using $\mathrm{TH}$ immunostaining to delineate VTA (Fig. 6A,D). Further, in a separate group of mice with PPTg implants and assessed by c-Fos (Fig. 4), we found a significant positive relationship between the number of nosepokes made and the number of c-Fos-labeled cells in the VTA of ChR2-expressing animals (Pearson, $p<0.05, r^{2}=$ $0.87, n=5$ ). There was no significant relationship between nosepokes and c-Fos expression in the VTA of mCherryexpressing animals (Pearson, $p=0.17, r^{2}=0.52, n=5$ ). Together, these results demonstrate that activation of PPTg glutmate projections to VTA is sufficient to promote behavioral reinforcement in mice. 
A

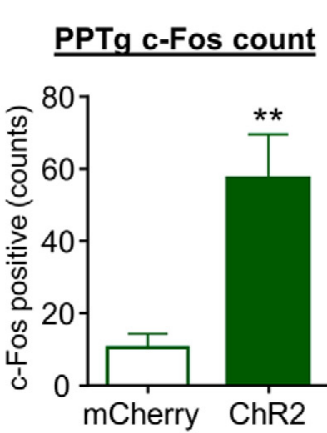

B

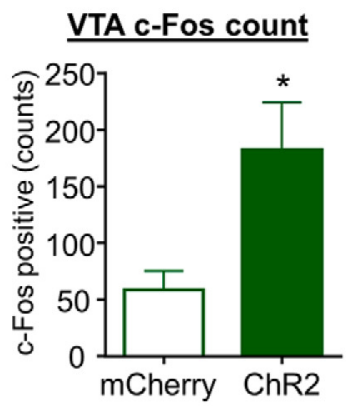

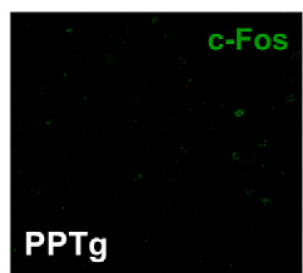
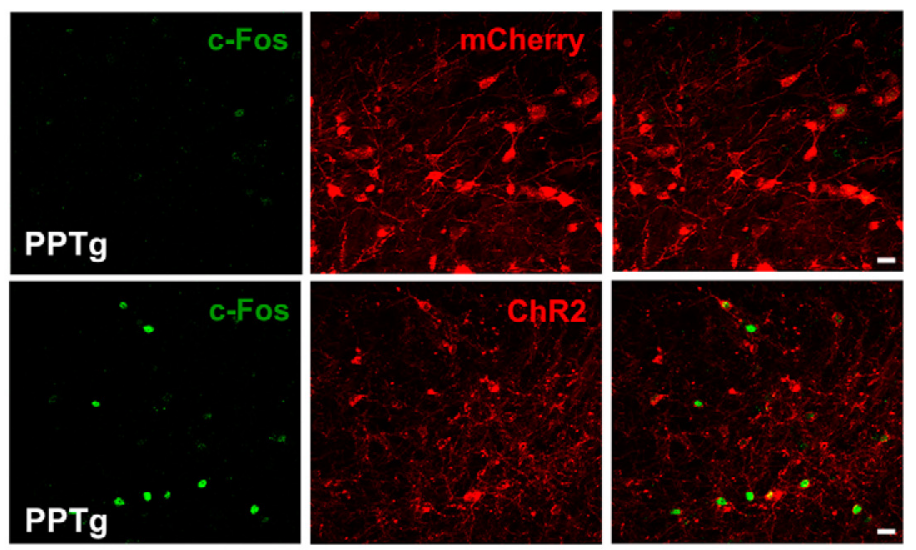

C
VTA c-Fos and TH

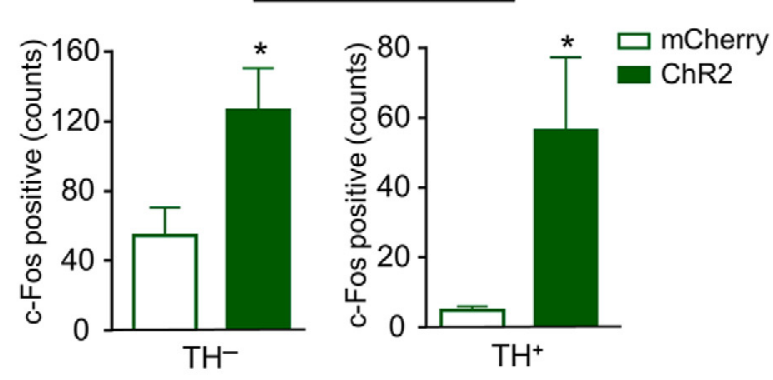

D
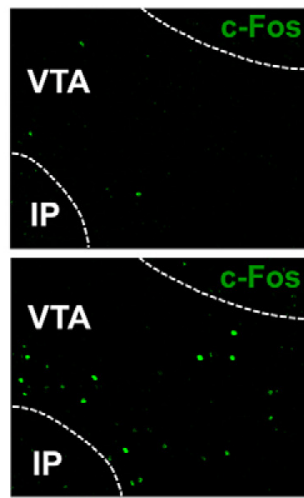
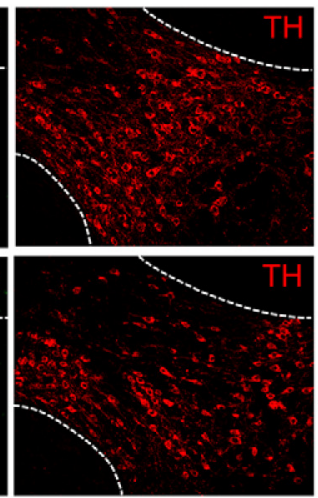
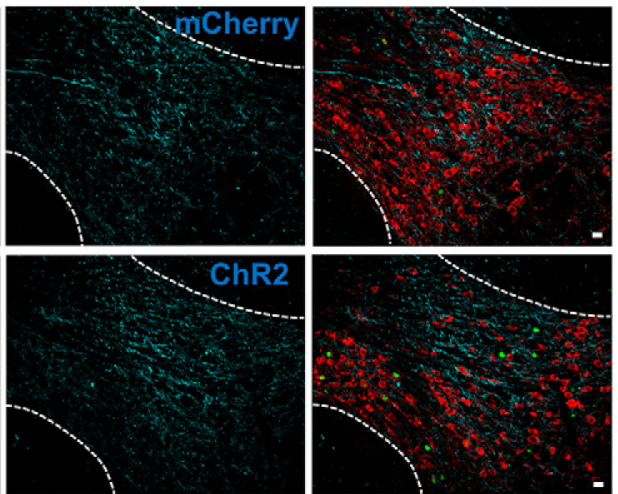

Figure 4. Optogenetic stimulation of PPTg glutamate neurons induces c-Fos in PPTg and VTA dopamine neurons. A, Following the in vivo photostimulation of PPTg glutamate neurons, the number of c-Fos ${ }^{+}$nuclei is increased in the PPTg of mice expressing ChR2:mcherry compared with mCherry controls. ${ }^{* *} p<0.01$ (unpaired $t$ test). $n=5$ mice. Representative images through PPTg. Scale bar, $20 \mu \mathrm{m} . \boldsymbol{B}$, In vivo photostimulation of PPTg glutamate neurons also increased number of c-Fos ${ }^{+}$neurons in the VTA and $(\boldsymbol{C})$ in both $\mathrm{TH}^{+}$and $\mathrm{TH}^{-}$cells. ${ }^{*} p<0.05$ (unpaired $t$ test). $n=5$ mice. $\boldsymbol{D}$, Representative images through VTA. IP, interpeduncular nucleus. Scale bar, $20 \mu \mathrm{m}$.

\section{Discussion}

The VTA contains heterogeneous cell types and receives projections from many brain areas, including ascending inputs from mesopontine regions (Phillipson, 1979; Fields et al., 2007; Faget et al., 2016). Two mesopontine regions enriched in cholinergic cells, the PPTg and LDTg, send both cholinergic and glutamatergic projections to the VTA (Geisler et al., 2007; Martinez-Gonzalez et al., 2011). However, the ubiquity of glutamate-mediated neurotransmission constrains the interpretation of pharmacological, lesion, or electrical stimulation based approached to understanding the function of discrete transmitter-defined neural circuits, particularly within heterogeneous brain regions, such as the PPTg and VTA. Here we take advantage of optogenetic methods to demonstrate that the glutamatergic component of the PPTg to VTA circuit can drive positive behavioral reinforcement using operant assays. We further show that the PPTg inputs target both dopamine and nondopamine neu- rons in the VTA and provide evidence that the excitatory impact on dopamine neurons may be greater. This study thus extends previous works that have shown that PPTg inputs to the VTA can drive dopamine release in vitro (Good and Lupica, 2010) and in vivo (Dautan et al., 2016) to demonstrate that the glutamatergic component of the PPTg input to VTA preferentially targets dopamine neurons and can serve as a positive reinforcer.

Recent studies have used optogenetics to demonstrate that PPTg cholinergic neurons can modulate behavioral reinforcement. In an instrumental lever-pressing assay for sugar pellets, photostimulation of PPTg cholinergic neurons reduced the rate of extinction in rats (Dautan et al., 2016). In another study, optogenetic stimulation of PPTg cholinergic projections in rat VTA produced a conditioned place preference, whereas their inhibition produced conditioned place avoidance (Xiao et al., 2016). Here we showed that VGLUT2 ${ }^{+}$ PPTg neurons project bilaterally to VTA where they make functional 
A

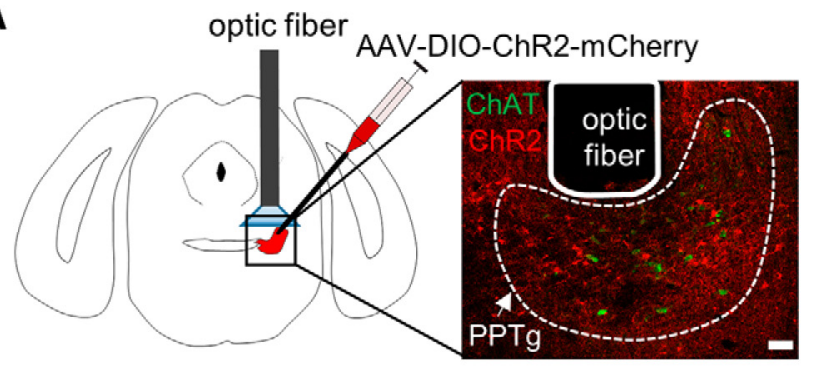

B

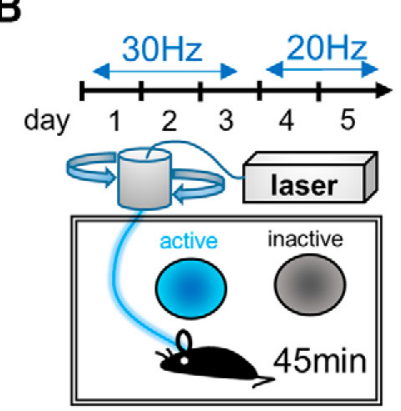

C

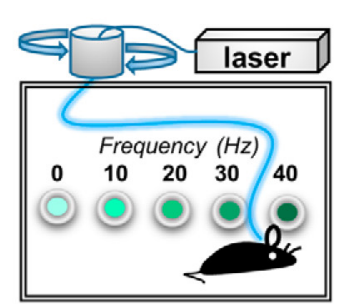

D

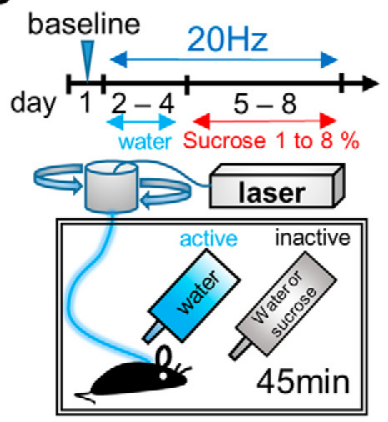

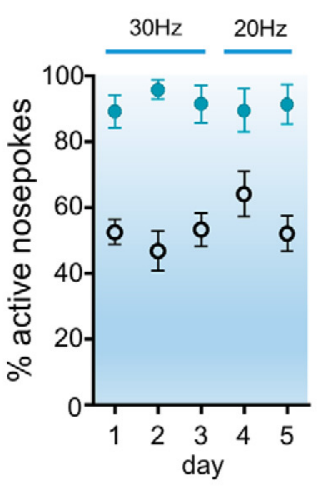
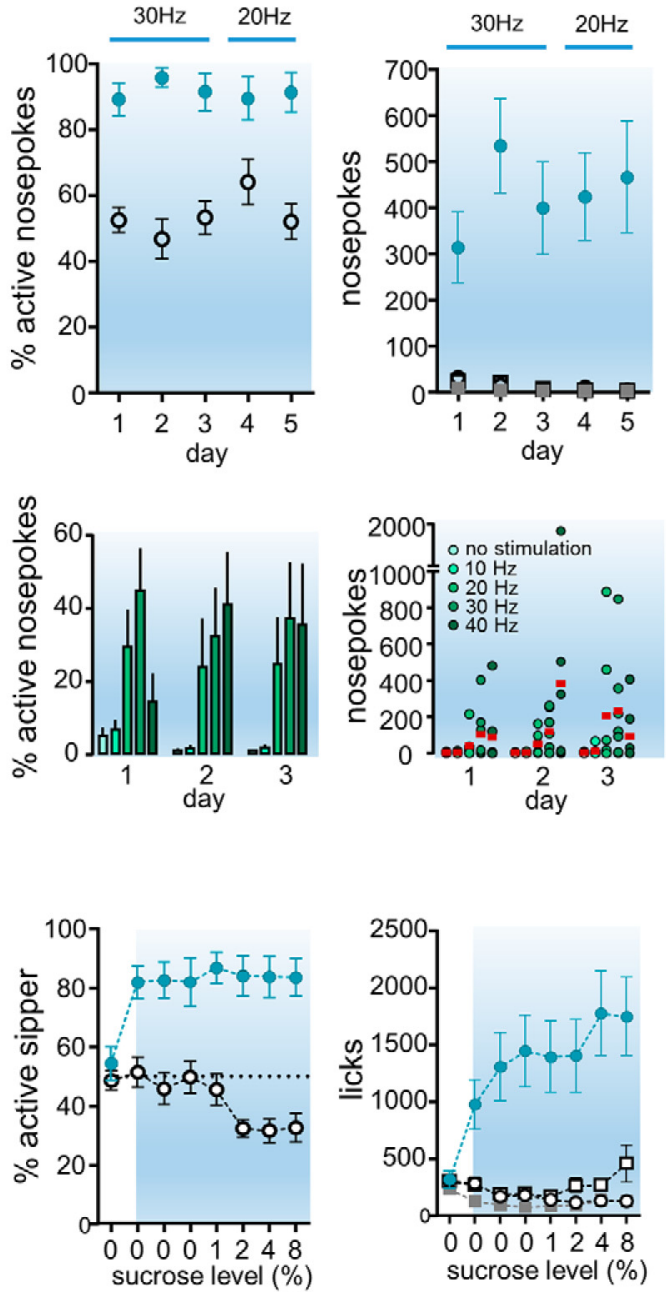

E

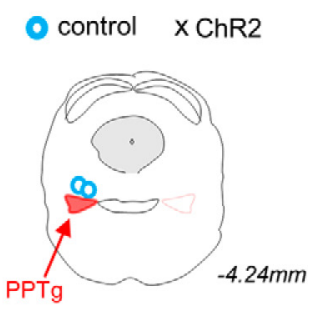

- mCherry active

- ChR2 inactive

口 mCherry inactive
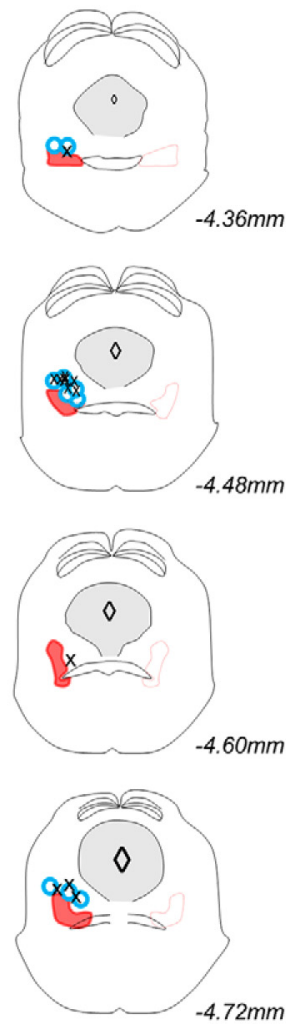

Figure 5. Activation of glutamate neurons in the PPTg promotes behavioral reinforcement. $A$, Schematic of optogenetic stimulation targeting glutamate neurons in PPTg. Example image showing optic fiber location relative to ChR2:mCherry expression. Scale bar, $100 \mu \mathrm{m}$. B, Schematic of the two-nosepoke intracranial self-stimulation task. ChR2-expressing mice $(n=13)$, but not control mice $(n=12)$, develop a preference for the active nosepoke (treatment, $\left.F_{(1,23)}=49.9, p<0.001\right)$ and a greater number of active nosepokes (treatment, $F_{(1,46)}=21.6, p<0.001$; nosepoke, $F_{(1,46)}=23.5, p<0.001$; treatment $\times$ nosepoke, $\left.F_{(1,46)}=23.2, p<0.001\right)$. Data are mean \pm SEM. C, Schematic illustrating 5-nosepoke discrimination task where responding on the active nosepoke triggers $0.5 \mathrm{~s}$ photostimulation of ChR2 in the PPTg of VGLUT2 ${ }^{\text {(re }}$ mice at various frequencies $(0,10,20,30$, and $40 \mathrm{~Hz})$. Photostimulation of VGLUT2 ${ }^{+}$PPTg neurons led to a preference for faster frequency stimulation (frequency, $F_{(4,30)}=9.4, p<0.001$ ). Data are mean \pm SEM. Number of nosepokes made on each hole. Dots represent individual data points. Horizontal bars represent means. $\boldsymbol{D}$, Schematic of the two bottle choice task. ChR2-expressing mice $(n=13)$ display an immediate preference for the active sipper, whereas control mice $(n=12)$ show no initial preference. Control mice develop a preference for escalating concentration of sucrose (inactive sipper), whereas ChR2-expressing mice show a sustained preference for photostimulation (treatment, $F_{(1,23)}=32.7, p<0.001$; day, $F_{(7,161)}=4.9, p<0.001$; day $\times$ treatment, $\left.F_{(7,161)}=9.9, p<0.001\right)$ and make more active licks (treatment, $F_{(1,46)}=12.0, p<0.01 ;$ sipper, $F_{(1,46)}=15.1$, $p<0.001$; day, $F_{(7,322)}=6.0, p<0.001$; day $\times$ treatment $\times$ sipper, $\left.F_{(7,322)}=12.8, p<0.001\right)$. Data are mean \pm SEM. $E$, Optic fiber placements in the PPTg.

glutamatergic synapses. But only very few ChR2-labeled VGLUT2 ${ }^{\text {Cre }}$ PPTg neurons coexpressed the cholinergic marker ChAT when AAVs were used to drive transgene expression, suggesting that the behavioral effects we observed with optogenetic stimulation relied principally on glutamate release.

The mesopontine tegmentum contains glutamate neurons that are involved in various brain functions, including motiva- tion, reward, and locomotion (Maskos, 2008; Ryczko and Dubuc, 2013; Gut and Winn, 2016). PPTg lesions can interfere with reward learning in conditioned reinforcement tasks (Inglis et al., 2000) and severely blunt the reinforcing effects of opiates and psychostimulants as assessed by conditioned place preference or self-administration (Bechara and van der Kooy, 1989; Olmstead et al., 1998). Further, 45\% of PPTg neurons were shown sensitive 

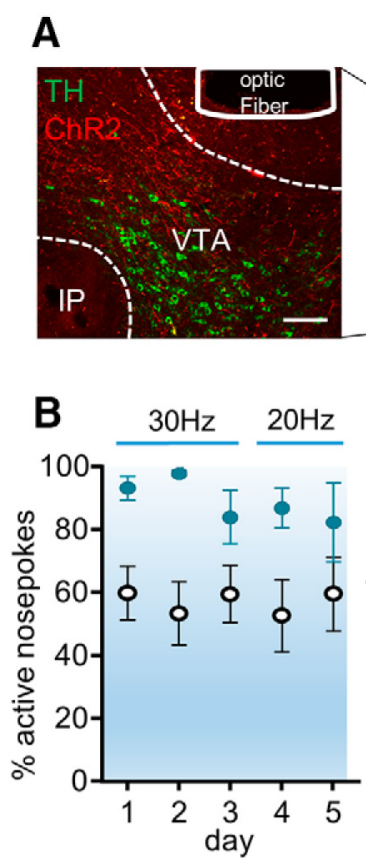

D

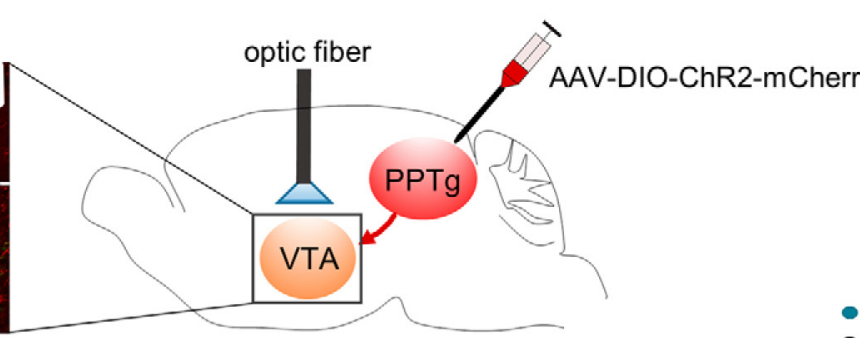

- ChR2 active

- mCherry active

- ChR2 inactive

ㅁ mCherry inactive

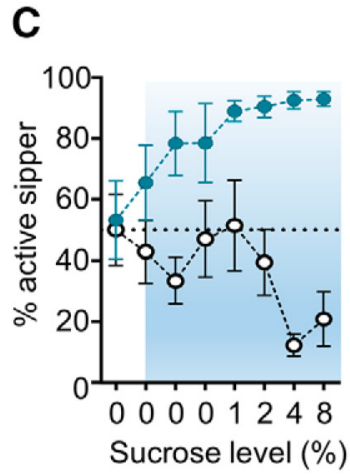

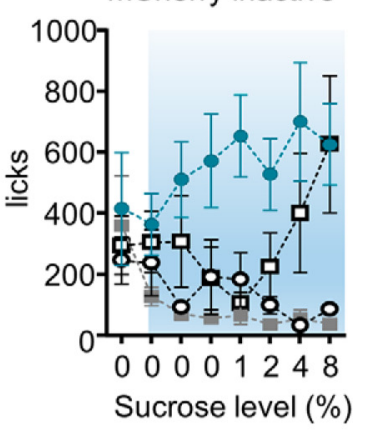

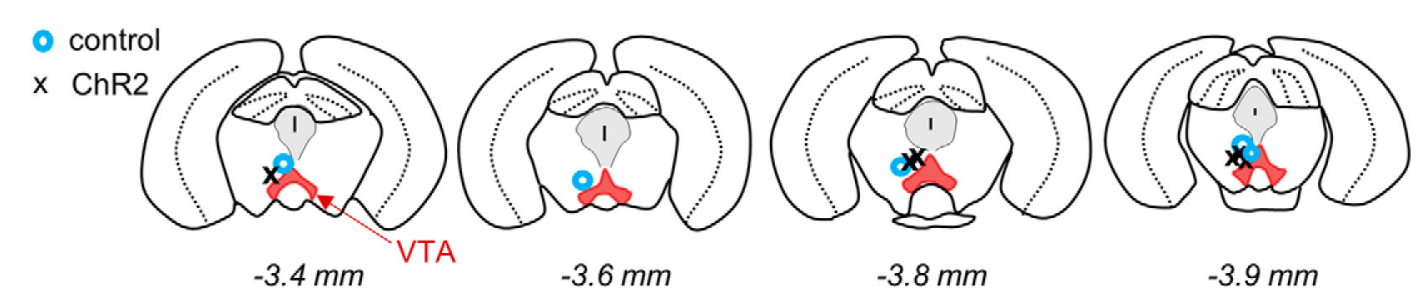

Figure 6. Photostimulation of PPTg glutamate terminals in the VTA can serve as a reinforcer. $\boldsymbol{A}$, Schematic of optogenetic stimulation targeting PPTg glutamate terminals in the VTA. Example image showing optic fiber location relative to ChR2:mCherry expression. Scale bar, $100 \mu \mathrm{m}$. $B$, ChR2-expressing mice $(n=6)$, but not control mice $(n=5)$, develop a strong preference for the active nosepoke (treatment, $F_{(1,9)}=12.2, p<0.01$ ) and poke more on the active side (nosepoke, $F_{(1,18)}=4.8, p<0.05 ;$ day, $F_{(4,72)}=2.8, p<0.05 ;$ treatment $\times$ nosepoke, $F_{(1,18)}=4.7, p<0.05$ ). Data are mean \pm SEM. $C$, ChR2-expressing mice $(n=6)$ display a preference for the active sipper, whereas control mice $(n=5)$ show no initial preference. Control mice develop a preference for escalating concentrations of sucrose (inactive sipper), whereas ChR2-expressing mice show a sustained preference for photostimulation (treatment, $F_{(1,9)}=35.5, p<0.001$; day $\times$ treatment, $\left.F_{(7,63)}=4.2, p<0.001\right)$ and more active licks (expression $\times$ sipper, $F_{(1,18)}=19.2, p<0.001$; day $\times$ treatment $\times$ sipper, $\left.F_{(7,126)}=3.2, p<0.01\right)$. Data are mean \pm SEM. $D, 0$ ptic fiber placements in the PPTg.

to reward acquisition (most were excited) (Norton et al., 2011), and pharmacological inactivation of the PPTg reduced conditioned dopamine responses (Pan and Hyland, 2005). Indeed, PPTg stimulation can induce burst firing in dopamine neurons (Lokwan et al., 1999; Floresco et al., 2003) and trigger dopamine release in the nucleus accumbens (Forster and Blaha, 2003). More recently, excitatory input from the LDTg and cholinergic input from PPTg have been shown sufficient to induce place preference (Lammel et al., 2012; Xiao et al., 2016). Together, these data point toward a role for excitatory and potentially glutamate inputs from PPTg to VTA in supporting behavioral reinforcement, but this had not been tested directly.

We report here that photostimulation of $\mathrm{VGLUT}^{+}{ }^{+} \mathrm{PPTg}$ neurons can induce robust intracranial self-stimulation using several behavioral assays. Reinforcement was observed for stimulation frequencies of $20-40 \mathrm{~Hz}$, and self-stimulation was insensitive to sucrose competition by two-bottle choice. Evidence suggests that PPTg neurons targeting VTA may represent a distinct population, at least from those projecting to thalamic targets (Holmstrand and Sesack, 2011). Thus we tested and confirmed that activation of VGLUT2 ${ }^{+}$PPTg afferents to VTA was also sufficient to drive reinforcement.
We found that the majority of cells in the VTA were responsive to and excited by PPTg input. However, a subset of cells showed very small or undetectable EPSCs. To test whether the inputs may preferentially target VTA dopamine neurons, we included LY in the patch pipette and performed post hoc immunolabeling for $\mathrm{TH}$. Using this approach provides high certainty for positively identified TH cells, although there is the potential for soluble TH to dialyze from the cell before fixation (Zhang et al., 2010), which could lead to false negatives. Nonetheless, our data are consistent with the hypothesis that both dopamine and nondopamine neurons were excited by PPTg inputs, consistent with our recent work showing that PPTg inputs do not selectively target VTA dopamine over GABA or glutamate neurons (Faget et al., 2016). However, we found that identified dopamine neurons yielded significantly larger EPSC amplitudes following single pulse photostimulation of PPTg inputs, suggesting that the synaptic strength or incidence of inputs from PPTg is greater on to $\mathrm{TH}^{+}$cells. In addition, our recordings were biased toward more lateral VTA regions that were more richly innervated by ChR2: $\mathrm{mCherry}^{+}$fibers from PPTg (medial VTA regions were considerably less fluorescent) and the majority of cells we filled (13 of 22) did colabel for TH. In line with these results, the in vivo 
optogenetic stimulation of PPTg glutamate neurons appeared to preferentially increase c-Fos expression in VTA dopamine neurons. We also examined the $\mathrm{SNc}$, which could also contribute to the observed behavioral reinforcement (Ilango et al., 2014), but found a relatively small number of c-Fos-positive neurons were activated compared with VTA (VTA-ctrl, $59 \pm 17$; VTA-ChR2, $183 \pm 41$; SNc-ctrl, $6 \pm 2$; SNc-ChR2, $26 \pm 8$ ). Although these data do not rule out contributions for nondopamine VTA neurons or PPTg inputs to regions other than VTA in the observed behavioral responses, the data do demonstrate that PPTg inputs directly excite VTA dopamine neurons and that activation of this projection can elicit behavioral reinforcement.

\section{References}

Bechara A, van der Kooy D (1989) The tegmental pedunculopontine nucleus: a brain-stem output of the limbic system critical for the conditioned place preferences produced by morphine and amphetamine. J Neurosci 9:3400-3409. Medline

Charara A, Smith Y, Parent A (1996) Glutamatergic inputs from the pedunculopontine nucleus to midbrain dopaminergic neurons in primates: Phaseolus vulgaris-leucoagglutinin anterograde labeling combined with postembedding glutamate and GABA immunohistochemistry. J Comp Neurol 364:254-266. CrossRef Medline

Dautan D, Souza AS, Huerta-Ocampo I, Valencia M, Assous M, Witten IB, Deisseroth K, Tepper JM, Bolam JP, Gerdjikov TV, Mena-Segovia J (2016) Segregated cholinergic transmission modulates dopamine neurons integrated in distinct functional circuits. Nat Neurosci 19:10251033. CrossRef Medline

Faget L, Osakada F, Duan J, Ressler R, Johnson AB, Proudfoot JA, Yoo JH, Callaway EM, Hnasko TS (2016) Afferent inputs to neurotransmitterdefined cell types in the ventral tegmental area. Cell Rep 15:2796-2808. CrossRef Medline

Fields HL, Hjelmstad GO, Margolis EB, Nicola SM (2007) Ventral tegmental area neurons in learned appetitive behavior and positive reinforcement. Annu Rev Neurosci 30:289-316. CrossRef Medline

Floresco SB, West AR, Ash B, Moore H, Grace AA (2003) Afferent modulation of dopamine neuron firing differentially regulates tonic and phasic dopamine transmission. Nat Neurosci 6:968-973. CrossRef Medline

Forster GL, Blaha CD (2003) Pedunculopontine tegmental stimulation evokes striatal dopamine efflux by activation of acetylcholine and glutamate receptors in the midbrain and pons of the rat. Eur J Neurosci 17: 751-762. CrossRef Medline

Futami T, Takakusaki K, Kitai ST (1995) Glutamatergic and cholinergic inputs from the pedunculopontine tegmental nucleus to dopamine neurons in the substantia nigra pars compacta. Neurosci Res 21:331-342. CrossRef Medline

Geisler S, Wise RA (2008) Functional implications of glutamatergic projections to the ventral tegmental area. Rev Neurosci 19:227-244. CrossRef Medline

Geisler S, Derst C, Veh RW, Zahm DS (2007) Glutamatergic afferents of the ventral tegmental area in the rat. J Neurosci 27:5730-5743. CrossRef Medline

Good CH, Lupica CR (2010) Afferent-specific AMPA receptor subunit composition and regulation of synaptic plasticity in midbrain dopamine neurons by abused drugs. J Neurosci 30:7900-7909. CrossRef Medline

Grace AA, Floresco SB, Goto Y, Lodge DJ (2007) Regulation of firing of dopaminergic neurons and control of goal-directed behaviors. Trends Neurosci 30:220-227. CrossRef Medline

Gut NK, Winn P (2016) The pedunculopontine tegmental nucleus: a functional hypothesis from the comparative literature. Mov Disord 31:615624. CrossRef Medline

Holmstrand EC, Sesack SR (2011) Projections from the rat pedunculopontine and laterodorsal tegmental nuclei to the anterior thalamus and ventral tegmental area arise from largely separate populations of neurons. Brain Struct Funct 216:331-345. CrossRef Medline
Ilango A, Kesner AJ, Keller KL, Stuber GD, Bonci A, Ikemoto S (2014) Similar roles of substantia nigra and ventral tegmental dopamine neurons in reward and aversion. J Neurosci 34:817-822. CrossRef Medline

Inglis WL, Olmstead MC, Robbins TW (2000) Pedunculopontine tegmental nucleus lesions impair stimulus-reward learning in autoshaping and conditioned reinforcement paradigms. Behav Neurosci 114:285-294. CrossRef Medline

Lammel S, Lim BK, Ran C, Huang KW, Betley MJ, Tye KM, Deisseroth K, Malenka RC (2012) Input-specific control of reward and aversion in the ventral tegmental area. Nature 491:212-217. CrossRef Medline

Lokwan SJ, Overton PG, Berry MS, Clark D (1999) Stimulation of the pedunculopontine tegmental nucleus in the rat produces burst firing in A9 dopaminergic neurons. Neuroscience 92:245-254. CrossRef Medline

Lüscher C, Malenka RC (2011) Drug-evoked synaptic plasticity in addiction: from molecular changes to circuit remodeling. Neuron 69:650-663. CrossRef Medline

Martinez-Gonzalez C, Bolam JP, Mena-Segovia J (2011) Topographical organization of the pedunculopontine nucleus. Front Neuroanat 5:22. CrossRef Medline

Maskos U (2008) The cholinergic mesopontine tegmentum is a relatively neglected nicotinic master modulator of the dopaminergic system: relevance to drugs of abuse and pathology. Br J Pharmacol 153 [Suppl 1]:S438-S445.

Mena-Segovia J, Bolam JP, Magill PJ (2004) Pedunculopontine nucleus and basal ganglia: distant relatives or part of the same family? Trends Neurosci 27:585-588. CrossRef Medline

Norton AB, Jo YS, Clark EW, Taylor CA, Mizumori SJ (2011) Independent neural coding of reward and movement by pedunculopontine tegmental nucleus neurons in freely navigating rats. Eur J Neurosci 33:1885-1896. CrossRef Medline

Okada K, Toyama K, Inoue Y, Isa T, Kobayashi Y (2009) Different pedunculopontine tegmental neurons signal predicted and actual task rewards. J Neurosci 29:4858-4870. CrossRef Medline

Olmstead MC, Munn EM, Franklin KB, Wise RA (1998) Effects of pedunculopontine tegmental nucleus lesions on responding for intravenous heroin under different schedules of reinforcement. J Neurosci 18:50355044. Medline

Pan WX, Hyland BI (2005) Pedunculopontine tegmental nucleus controls conditioned responses of midbrain dopamine neurons in behaving rats. J Neurosci 25:4725-4732. CrossRef Medline

Phillipson OT (1979) Afferent projections to the ventral tegmental area of Tsai and interfascicular nucleus: a horseradish peroxidase study in the rat. J Comp Neurol 187:117-143. CrossRef Medline

Roseberry TK, Lee AM, Lalive AL, Wilbrecht L, Bonci A, Kreitzer AC (2016) Cell-type-specific control of brainstem locomotor circuits by basal ganglia. Cell 164:526-537. CrossRef Medline

Ryczko D, Dubuc R (2013) The multifunctional mesencephalic locomotor region. Curr Pharm Des 19:4448-4470. CrossRef Medline

Vong L, Ye C, Yang Z, Choi B, Chua S Jr, Lowell BB (2011) Leptin action on GABAergic neurons prevents obesity and reduces inhibitory tone to POMC neurons. Neuron 71:142-154. CrossRef Medline

Wang HL, Morales M (2009) Pedunculopontine and laterodorsal tegmental nuclei contain distinct populations of cholinergic, glutamatergic and GABAergic neurons in the rat. Eur J Neurosci 29:340-358. CrossRef Medline

Xiao C, Cho JR, Zhou C, Treweek JB, Chan K, McKinney SL, Yang B, Gradinaru V (2016) Cholinergic mesopontine signals govern locomotion and reward through dissociable midbrain pathways. Neuron 90:333-347. CrossRef Medline

Yau HJ, Wang DV, Tsou JH, Chuang YF, Chen BT, Deisseroth K, Ikemoto S, Bonci A (2016) Pontomesencephalic tegmental afferents to VTA nondopamine neurons are necessary for appetitive Pavlovian learning. Cell Rep 16:2699-2710. CrossRef Medline

Zhang TA, Placzek AN, Dani JA (2010) In vitro identification and electrophysiological characterization of dopamine neurons in the ventral tegmental area. Neuropharmacology 59:431-436. CrossRef Medline 